\title{
Siglen und Abkürzungen für Archivalien
}

Briefverzeichnis 1798/99 〈Verzeichnis Goethes über abgesandte Briefe Dezember 1798-Januar 1799.) GSA, Sign.: 28/24, Bl. 2

Cotta, Druckauftragsbuch I Druckauftragsbuch (1800-1810). SNM/DLA Marbach, Cotta-Archiv (Stiftung der Stuttgarter Zeitung), Sign.: Geschäftsbücher IV(20).

Färber-Calender 1799 Historischer Gespräch-Calender, zwischen Staps und Kasper 2. Bauern; Schwitzkasten ein Dorfbalbier; Wenzel und Superklug 2. Bürger; Klos ein Dorfwirth. Auf das Jahr nach der Geburt Christi, 1799. Jena, privilegirter Wertherischer Calender, zu haben bey Philipp Jacob Lorenz Werther 1798$\rangle$. 〈Darin handschriftliche Notizen des Besitzers Johann David Färber.) ThULB Jena, Sign.: Nachl. Martin q 20:15.

Färber-Calender $1800 \quad$ Neuer und verbesserter Historien-Calender, Auf das Jahr nach der Geburt Christi, 1800. Darinnen zu finden der Planeten-Lauf, Aspecten-Stand, und Finsternisse, Witterung, Pflanz- und Säetage, Sonnen Auf- und Untergang und alles, was zu einem vollständigen Calender nöthig ist. Jena, privilegirter Wertherischer Calender, im Verlag der Wertherischen Erben 〈1799〉. 〈Darin handschriftliche Notizen des Besitzers Johann David Färber.〉 ThULB Jena, Sign.: Nachl. Martin q 20:16.

FB 1799

Fourier-Buch / auf das Jahr / 1799. / dermalen geführet, / von den Hof-Fouriers, / Johann Christoph Waitz / und / August Christian Friedrich / Martini. (Fourierbuch zur Hofhaltung des Herzogs Carl August. 1. Jan. - 31. Dez. 1799). 175 Bl., pag. 1-349. LATh - HStA, Sign.: Hofmarschallamt, Nr 4548 .

FB 1800

Fourier-Buch / auf das Jahr / 1800. / geführet / von / den beyden Hof-Fouriers / Johann Christoph Waitz, / und / August Christian Friedrich Martini / in / Weimar. (Fourierbuch zur Hofhaltung des Herzogs Carl August. 1. Jan. - 31. Dez. 1800). 125 Bl., pag. 1-249. LATh-HStA, Sign.: Hofmarschallamt, $\mathrm{Nr} 4549$. 
GR/Belege 1799, 2

GR/Belege 1799, 3

GR/Belege 1799, 4

GR/Belege 1799, 5

GR/Belege 1800, 1

GR/Belege 1800, 2

GR/Belege 1800, 3

GR/Belege 1800, 4

GR/Belege 1801, 1

GR/Belege 1801, 5

GR/Jena 1799, 1

GR/Jena 1799, 2

GR/RB 1799, 1
Goethe. Rechnungen. Belege zur Einnahme- und Ausgabe-Rechnung November 1798 - April 1799. GSA, Sign.: 34/XIV,3,2.

Goethe. Rechnungen. Belege zur Einnahme- und Ausgabe-Rechnung April-Juni 1799. GSA, Sign.: 34/XIV,3,3.

Goethe. Rechnungen. Belege zur Einnahme- und Ausgabe-Rechnung März-Oktober 1799. GSA, Sign.: 34/XIV,3, 4.

Goethe. Rechnungen. Belege zur Einnahme- und Ausgabe-Rechnung Januar-Dezember 1799. GSA, Sign.: 34/XIV,3,5.

Goethe. Rechnungen. Belege zur Einnahme- und Ausgabe-Rechnung April 1799 - Januar 1800. GSA, Sign.: 34/XIV,6,1.

Goethe. Rechnungen. Belege zur Einnahme- und Ausgabe-Rechnung März-August 1800. GSA, Sign.: 34/XIV,6,2.

Goethe. Rechnungen. Belege zur Einnahme- und Ausgabe-Rechnung März-November 1800. GSA, Sign.: 34/XIV,6,3.

Goethe. Rechnungen. Belege zur Einnahme- und Ausgabe-Rechnung Januar-Dezember 1800. GSA, Sign.: 34/XIV,7.

Goethe. Rechnungen. Belege zur Einnahme- und Ausgabe-Rechnung Juli 1800 - Januar 1801. GSA, Sign.: 34/XV,3,1.

Goethe. Rechnungen. Belege zur Einnahme- und Ausgabe-Rechnung Dezember 1800 - Dezember 1801. GSA, Sign.: 34/XV,3,5.

Goethe. Rechnungen. Sonderrechnungen. Jenaische Reiserechnung. 7.-28. Februar 1799. GSA, Sign.: 34/XIV,2,1.

Goethe. Rechnungen. Sonderrechnungen. Jenaische Reiserechnung. 16. September-14. Oktober 1799. 10. November - 8. Dezember 1799. GSA, Sign.: 34/XIV,2,2.

Goethe. Rechnungen. Rechnungsbuch. Einnahme und Ausgabe Dezember 1798 - März 1799. GSA, Sign.: 34/XIV,1,1. 
$G R / R B$ 1799, 2

GR/RB 1799, 4

GR/RB 1800, 1

GR/RB 1800, 3

Knebel, Tgb. 1799

Knebel, Tgb. 1800

Knebel, Tgb. 1801

Knebel, Tgb. 1803

Theater/Musik Weimar
Goethe. Rechnungen. Rechnungsbuch. Einnahme und Ausgabe März-Juni 1799. GSA, Sign.: 34/XIV,1,2.

Goethe. Rechnungen. Rechnungsbuch. Einnahme und Ausgabe Oktober-Dezember 1799. GSA, Sign.: 34/XIV,1, 4.

Goethe. Rechnungen. Rechnungsbuch. Einnahme und Ausgabe Dezember 1799-März 1800. GSA, Sign.: 34/XIV,4,1.

Goethe. Rechnungen. Rechnungsbuch. Einnahme und Ausgabe Oktober-Dezember 1800. GSA, Sign.: 34/XIV,4,3.

Carl Ludwig von Knebel: Tagebuch 1799. GSA, Sign.: 54/375.

Carl Ludwig von Knebel: Tagebuch 1800. GSA, Sign.: 54/376.

Carl Ludwig von Knebel: Tagebuch 1801. GSA, Sign.: 54/377.

Carl Ludwig von Knebel: Tagebuch 1803. GSA, Sign.: 54/379.

Theater und Musik in Weimar 1754-1990. - Online verfügbar. 